\title{
Estimating energy requirements: regression based prediction equations or multiples of resting metabolic rate
}

\author{
Michael I Goran * \\ Institute for Prevention Research and Departments of Preventive Medicine and Physiology \& Biophysics, Keck School \\ of Medicine, University of Southern California, Los Angeles, CA, USA
}

\begin{abstract}
Energy requirements have traditionally been determined based on multiples of resting metabolic rate (RMR), known as Physical Activity Levels (PAL). With more data from doubly labelled water studies alternative approaches for estimating energy requirements have been suggested. Statistical analysis reveals that body weight explains more of the variance in total energy expenditure (TEE) than does RMR. The explanation for this phenomenon is that body weight contributes to the variance of both RMR and the other major determinant of TEE, i.e. physical activity related energy expenditure. Thus, in effect, the regression-based approach provides a more physiological appropriate model for TEE. Its major departure from tradition, difference from current adult proposals, and time taken for acceptance are the disadvantages of the regression-based approach.
\end{abstract}

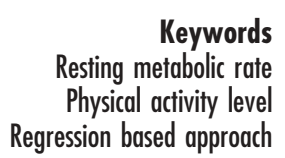

Resting metabolic rate Regression based approach

\section{Introduction}

Energy requirements have traditionally been determined based on multiples of resting metabolic rate (RMR), known as Physical Activity Levels (PAL). This approach was developed prior to the availability of doubly labelled water, which provides a more direct estimate of energy requirements as a measure of total energy expenditure (TEE). As more doubly labelled water data becomes available, alternative approaches for estimating energy requirements have been suggested. The purpose of this paper is to provide a comparison of advantages and disadvantages of these two approaches for estimating energy requirements (see Table 1).

\section{Regression based approach}

The regression based approach for estimating TEE has been suggested in various other studies ${ }^{1,2}$, as well as, in the analysis of data for infants ${ }^{3}$ and children ${ }^{4}$ for this consultation in this series of papers. This approach uses multiple regression techniques to develop prediction models of TEE as a function of measured predictor variables such as body weight, age, RMR, etc. The regression approach is a major departure from the convention approach of predicting energy requirements as multiples of RMR.

There are several advantages of the regression based approaches. The first major advantage is that this approach is evidence based and has been developed based on a pool of actual measures of TEE and potential predictor variables. It is worthy to note that both Butte ${ }^{3}$ and Torun ${ }^{4}$ worked independently to review existing data from infants and children, but yet derived a very similar approach for estimating total energy requirements using a simple, yet effective prediction model. Both prediction equations were based on simple available measures (body weight) and provided relatively accurate predictions of TEE. Interestingly, the data sets that have been used to define regression approaches for TEE are significantly larger than the data sets used elsewhere in this series of papers to derive prediction equations for RMR in children. Clearly further studies and analysis will be required to expand the data sets into more heterogeneous groups, as well as perform cross-validations of the proposed prediction equations on independent data sets.

The statistical analysis is revealing in that clearly body weight explains more of the variance in TEE than does RMR, as has been observed in other analysis where RMR typically only explains $\sim 50 \%$ of the variance in $\mathrm{TEE}^{5}$. The explanation for this phenomenon is that body weight contributes to the variance of both RMR and the other major determinant of TEE, i.e. physical activity related energy expenditure. Thus, in effect, the regression based approach provides a more physiological appropriate model for TEE, as compared to the PAL approach which assumes TEE is composed of multiples of RMR.

The disadvantages of the regression based approach are that it is a major departure from tradition, is different from current adult proposals, and will take time to be accepted. In addition, current equations are based mainly on weight (several also include height, age and gender), and may 
Table 1 Summary of strengths and limitations of the regression based approach and the PAL ratio approach for estimating energy requirements

\begin{tabular}{|c|c|c|}
\hline & Strengths & Limitations \\
\hline Regression based & $\begin{array}{l}\text { Evidence based (i.e. based on measures of TEE) } \\
\text { Several researchers have independently proposed } \\
\text { this new approach } \\
\text { Simple and practical to implement } \\
\text { Typically based on body weight which predicts } \\
\text { more of the variance in TEE than RMR } \\
\text { Physiologically sound } \\
\text { Statistically and mathematically sound }\end{array}$ & $\begin{array}{l}\text { Major departure from tradition } \\
\text { If used for children may not be consistent with adults } \\
\text { Current equations mainly limited to weight based } \\
\text { prediction and may need modification to specify activity }\end{array}$ \\
\hline PAL ratio approach & $\begin{array}{l}\text { Traditional approach } \\
\text { Commonly used and widely accepted }\end{array}$ & $\begin{array}{l}\text { Need to first predict RMR then apply PAL factor } \\
\text { 2-stage prediction may compound prediction error } \\
\text { Clumsy to apply } \\
\text { PALs are arbitrary and have not been specifically } \\
\text { developed for children and infants } \\
\text { Different PALs are not needed for infants due to limited } \\
\text { range of activity } \\
\text { PAL is highly variable, even within heterogeneous groups } \\
\text { PAL ratio violates mathematical assumption of non-zero } \\
\text { intercept } \\
\text { Not based on sound physiological model of TEE (should } \\
\text { be sum of components, not multiples of one component) } \\
\text { Assumes physical activity energy cost is dependent on the } \\
\text { same factors as for RMR }\end{array}$ \\
\hline
\end{tabular}

Abbreviations: PAL - Physical Activity Level; TEE - total energy expenditure; RMR - resting metabolic rate.

need to be modified with other variables to specify different activity levels. Nevertheless, since both the regression based approach and the PAL approach are based mainly on weight, a direct comparison of the two approaches can be easily obtained.

\section{PAL approach (multiples of RMR)}

The major advantage of the PAL approach is that it is a traditional and commonly used approach. On the surface

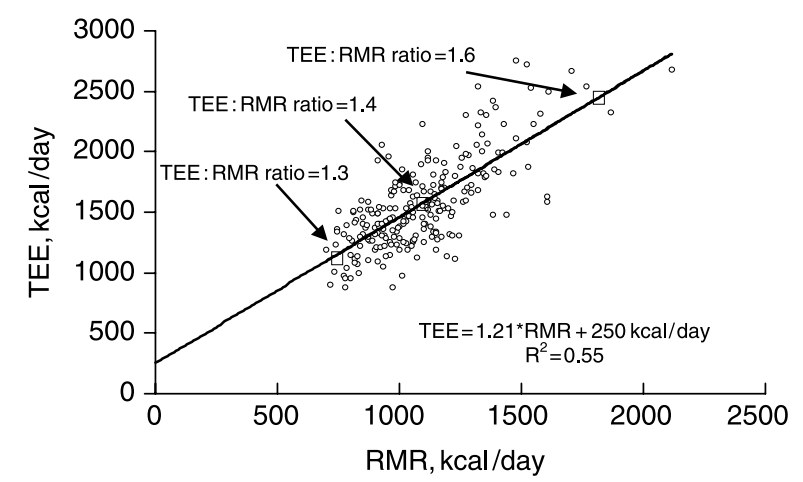

Fig. 1 Spurious ratios when comparing children at low, medium and high levels of total energy expenditure (TEE). Data from 231 children previously published ${ }^{2}$ showing a regression relationship between TEE and RMR. The three 'hypothetical' children are shown as large circles at low, medium and high levels of energy expenditure. All three children lie on the regression line and therefore have a similar total relative to resting energy expenditure, however, the TEE:RMR ratios are widely different. The difference in the TEE:RMR ratio is a spurious observation because this ratio fails to take into account the non-zero intercept in the relationship between total and resting energy expenditure this approach is simple to use but a closer analysis of this approach reveals several limitations.

First the PAL approach is actually awkward to use in that a two-step prediction is required that could potentially compound prediction error. In the first step, RMR is predicted using body weight based equations. The equations proposed are based on a limited data set for children and infants. In the second step a PAL is applied and the predicted RMR is multiplied by that factor to obtain an estimate of energy requirements. The PALs that are frequently used for light, moderate, and heavy physical activity are somewhat arbitrary, and were not developed specifically for use in children. In general, the PAL approach may not be statistically or mathematically appropriate because it is a ratio (PAL $=$ TEE:RMR). In other words the PAL model assumes a linear relationship between TEE and RMR that has a slope equivalent to PAL and a non-zero intercept. Several studies, including a meta-analysis ${ }^{6}$ reveal that the relationship between TEE and RMR is linear but has a variable slope, and more importantly, a variable and frequently non-zero intercept. The non-zero intercept is important since under this condition use of a constant PAL results in spurious differences when subjects are compared across the range of TEE levels (see Fig. 1). In an analysis of 574 measures of $\mathrm{TEE}^{7}$, the PAL index was validated by showing that it was uncorrelated with RMR, but it was not clear whether the TEE vs. RMR relationship satisfied the non-zero intercept requirement.

From a physiological perspective the assumption of multiples of RMR is also an inaccurate model of TEE, since TEE is composed of the sum of various components 
(RMR + activity + thermic effect of a meal) rather than multiples of one single component. Another physiological limitation is that applying PALs to RMR assumes that physical activity energy expenditure is dependent entirely on RMR, which is not the case. Moreover, since RMR is primarily weight dependent (or more specifically lean mass dependent), the PAL approach assumes that physical activity is primarily weight dependent. This may be true for some physical activities but unlikely to be true for all types and levels of physical activity.

\section{Summary and conclusion}

A summary of the strengths and limitations of the two approaches is provided in Table 1. The regression-based approach is recommended since it is evidence based, easier to apply and more statistically and physiologically appropriate. However, during the transition from the traditional PAL approach, it is relatively straightforward to make both approaches available so they can be compared.

\section{References}

1 Goran MI, Poehlman ET. Total energy expenditure and energy requirements in healthy elderly persons. Metabolism 1992; 41: 744-53.

2 Goran MI, Nagy TR, Gower BA, Mazariegos M, Solomons N, Hood V, Johnson R. Influence of sex, seasonality, ethnicity and geographic location on the components of total energy expenditure in young children: implications for energy requirements. American Journal of Clinical Nutrition 1998; 68(3): 675-82.

3 Butte N. Energy requirements of infants. Public Health Nutrition 2005; 8(7A): 953-67.

4 Torun B. Energy requirements of children and adolescents. Public Health Nutrition 2005; 8(7A): 968-93.

5 Goran MI. Variation in total energy expenditure in humans. Obesity Research 1995; 3: 59-66.

6 Carpenter WH, Poehlman ET, O'Connell M, Goran MI Influence of body composition and resting metabolic rate on variation in total energy expenditure: a meta analysis. American Journal of Clinical Nutrition 1995; 61: 4-10.

7 Black AE, Coward WA, Cole TJ, Prentice AM. Human energy expenditure in affluent societies: an analysis of 574 doubly labelled water measurements. European Journal of Clinical Nutrition 1996; 50: 72-92. 